\title{
Action d'un dérivé chloré de la Salicylanilide en milieu tropical, sur les Trématodes, parasites des bovins
}

\author{
par J. GUILHON $\left(^{*}\right)$ et M. GRABER (**)
}

\begin{abstract}
RESUME
L'oxyclozanide (pentachloro $3,3^{\prime}, 5,5$, 6 dihydroxy $2,2^{\prime}$ salicylanilide) administré per os, à des zébus des deux sexes, à Fort-Lamy (Tchad) s'est révélé actif contre Fasciola gigantica adulte et les Paramphistomidés de la panse à la dose unique de 7,5, ou mieux de 10 à $15 \mathrm{mg} / \mathrm{kg}$.

Pour détruire les fascioles immatures de 6 à 7 semaines, la dose de $40 \mathrm{mg} / \mathrm{kg}$ est encore insuffisante.

Ce corps n'a aucune activité à l'égard de Schistosoma bovis et de Dicrocoelium hospes. $75 \mathrm{mg} / \mathrm{kg}$.

Enfin, des accidents mortels apparaissent aux doses d'environ 60 à
\end{abstract}

\section{INTRODUCTION}

Dans le programme de recherches prévues pour apprécier, au Tchad, les propriétés de divers corps proposés pour lutter contre les principaux agents des distomoses des animaux domestiques (Fascioles, Paramphistomes, Schistosomes), l'oxyclozanide, au même titre que le Bromophénophos et le Nitroxynil, étudiés antérieurement, a retenu notre attention.

L'oxyclozanide, premier dérivé de la salicylanilide utilisé contre les fascioles est le pentachloro $3,3^{\prime}, 5,5^{\prime}, 6$ dihydroxy, 2, 2' salicylanilide.

Il se présente sous l'aspect d'une poudre blanche, cristalline insoluble dans l'eau, mais soluble dans l'alcool, l'acétone, le toluène et le xylène chauds. Il est doué d'une assez grande stabilité qui favorise une bonne conservation, quel que soit le lieu de son utilisation. Il est

(*) Laboratoire de Parasitologie, Ecole Nationale Vétérinaire, 94 Alfort.

(**) Laboratoire de Farcha, Fort-Lamy (Tchad); Chaire de Parasitologie, Ecole Nationale Vétérinaire de Lyon. livré, pour l'emploi, sous la forme d'une suspension aqueuse, blanchâtre, renfermant 3,4 p. 100 de principe actif $\left({ }^{1}\right)$.

Les premiers travaux concernant l'oxyclozanide ont été publiés en 1966. BROOME et JONES, chez des rats et des souris, artificiellement infestés avec des métacercaires de Fasciola hepatica, comparent le pouvoir antidistomien de divers corps (tétrachlorure de carbone, Hexachloréthane, tétrachlorodifluoroéthane, Hétol $\left({ }^{3}\right)$, Diaphène $\left({ }^{3}\right)$, Hexachlorophène $\left({ }^{4}\right)$ et oxyclozanide). Les quatre premiers n'ont que peu d'effets alors que les trois derniers sont actifs à des degrés divers (tableau I).

Les essais ont été effectués chez le bœuf, en Angleterre par WALLEY, VAUGHAN, JONES, KELSEY (1966), puis par SINCLAIR

(1) Ce corps est encore désigné dans le commerce par les termes de Zanil (I C I) et de Diplin (C E L A).

(2) 1,4 bis trichloro méthyl-benzol.

(3) Diaphène, Hilomid, Mitényl (3 parties de tribromo $3,4^{\prime}, 5$ salicylanilide et 1 partie de dibromo 4', 5 salicylanilide).

(4) Hexachloro 3, 5, 6- 3', 5', 6'-dihydroxy 2-2'-diphényl-méthane. 
TABLEAU N ${ }^{\circ}$ I

\begin{tabular}{|c|c|c|c|}
\hline \multirow{2}{*}{ Médicament } & \multicolumn{2}{|c|}{$\begin{array}{c}\text { Dose minimale active } \\
\mathrm{mg} / \mathrm{kg}\end{array}$} & \multirow{2}{*}{$\begin{array}{c}\text { Toxicité } \\
\mathrm{DL}_{50} \\
\text { (rats en mg } / \mathrm{kg} \text { ) }\end{array}$} \\
\hline & $\begin{array}{l}\text { Douves adu1tes } \\
\text { (rats) }\end{array}$ & $\begin{array}{c}\text { Douves immatures } \\
\text { (souris) }\end{array}$ & \\
\hline Diaphène & 100 & $200 \times 2$ & 600 \\
\hline Hexachlorophène & 10 & $100 \times 1$ & 100 \\
\hline Oxyclozanide & 15 & $100 \times 3$ & 1.000 \\
\hline
\end{tabular}

(1968) et FROYD (1968-1969); en Australie par BORAY et collab. (1967-1968); en Allemagne par REUSS et BROZEIT (1968); en Suède par HEDNER (1968); en Tanzanie par HILDEBRANDT et ILMOLELIAN (1968) et enfin en Pologne par TARCZYNSKI et collab. (1969).

Le but du présent travail est d'indiquer les résultats obtenus, à Fort-Lamy (République du Tchad), sur un petit nombre de zébus, naturellement ou expérimentalement infestés par Fasciola gigantica.

\section{MATERIEL ET METHODE}

\section{A. Animaux d'expérience}

Durant l'expérimentation 30 zébus ont été utilisés : 11 femelles âgées ( $\left.{ }^{5}\right)$ de réforme, originaires de la région de Massakory et 19 bouvillons, dont 7 achetés à Bouar en R.C.A.

Ils ont été répartis comme ci-après (tableau II).

\begin{tabular}{|c|c|c|c|}
\hline & $\begin{array}{l}\text { Femelles } \\
\text { âgêes }\end{array}$ & Bouvillons & Totaux \\
\hline $\begin{array}{l}\text { Essa1s thérapeutiques } \\
\text { sur les fascioles adultes }\end{array}$ & 7 & 4 & 11 \\
\hline $\begin{array}{l}\text { Essai sur Fasciola } \\
\text { gigontica inmatures }\end{array}$ & & 7 & 7 \\
\hline Témoins & 4 & 6 & 10 \\
\hline Essais de toxictté & & 2 & 2 \\
\hline
\end{tabular}

L'état des animaux était, dans l'ensemble, assez médiocre (sauf celui des bouvillons venus de Bouar), ce qui est normal, compte tenu de l'époque où les essais ont été effectués (printemps 1968 et 1969).

Les jeunes pesaient de 85 à $150 \mathrm{~kg}$ et les adultes de 153 à $296 \mathrm{~kg}$ (moyenne $248 \mathrm{~kg}$ ).

\section{B. Protocole expérimental}

Il fut identique à celui qui a été décrit lors des essais précédents concernant le Nitroxynil et le Bromophénophos.

\section{RESULTATS}

A. Témoins (tableau III)

Printemps 1968 (série A): 6 bouvillons (Bouar).

Printemps 1969 (série B) : 4 femelles âgées (Massakory).

(i) Métis de bovins «Kouris» et de zébus « arabes ». 
TABLEAU $\mathrm{N}^{\circ}$ III

\begin{tabular}{|c|c|c|c|c|c|}
\hline \multirow{2}{*}{ Helminthes obscrvés } & \multicolumn{2}{|c|}{$\begin{array}{l}\text { Nombre d'animaux } \\
\text { parasités }\end{array}$} & \multicolumn{3}{|c|}{$\begin{array}{l}\text { Poids ( } 1 \text { ) ou nombre } \\
\text { de parasites (moyenne) }\end{array}$} \\
\hline & Série A & Sêrie $\mathbf{B}$ & Série A & Série & $B$ \\
\hline Dicrocoetizon hospes & 4 & - & 5,5 & - & \\
\hline Fasoiola gigantica & 5 & 3 & 114 & 2 & \\
\hline CotyZophoron cotylophorum & 3 & - & 1 & - & \\
\hline Paramphis tomu microbothrium & - & 4 & - & $58 \mathrm{~g}$ & \\
\hline Paramphistomum sp. & - & 2 & - & $50 \mathrm{~g}$ & \\
\hline Schistosoma bovis & - & 3 & - & 16 & \\
\hline Thysaniezia ovitla & - & 1 & - & $1 \mathrm{~g}$ & \\
\hline Bosicola radiatum & - & 2 & - & 85 & \\
\hline Bunos tormm phlebotomum & 1 & - & 22 & - & \\
\hline Cooperia punctata & - & 2 & - & 2571 & \\
\hline Cooperia pectinata & - & 1 & - & 5000 & \\
\hline Cooperia sp. & 1 & - & 2400 & & \\
\hline Haemonous contortus & - & 1 & $\rightarrow$ & 300 & \\
\hline Artionema labiato-papiztoca & - & 2 & - & $\mathrm{L}$ & \\
\hline Onchocerea gutturos $\alpha$ & - & 2 & - & - & \\
\hline onchccerca armzzata & - & 2 & - & - & \\
\hline Buckleytais globutosa & 1 & 2 & - & 20 & \\
\hline
\end{tabular}

\section{B. Action sur les Trématodes}

1. Dicrocoelium hospes (canaux biliaires)

A la dose de $15 \mathrm{mg} / \mathrm{kg}$, l'oxyclozanide n'a aucune action sur Dicrocoelium hospes. Les voies biliaires des zébus traités renfermaient plus de petites douves vivantes (17) que celles des sujets témoins $(5,5)$.

\section{Fasciola gigantica immature}

(parenchyme hépatique et voies biliaires)

L'action du médicament a été appréciée sur
7 bouvillons infestés, expérimentalement, avec des métacercaires de Fasciola gigantica. Six d'entre eux en ont ingéré 500 et le septième 5.000 en deux temps. Les six premiers ont été traités 42,54 et 72 jours après l'infestation avec une dose unique comprise entre 10 et $40 \mathrm{mg} / \mathrm{kg}$, alors que le dernier a reçu une seule dose de $15 \mathrm{mg} / \mathrm{kg} 85$ jours après la dernière jnfestation.

Les animaux ont été sacrifiés 7 jours après l'administration du douvicide. Les résultats sont indiqués dans le tableau IV.

TABIEAL ::" IV

\begin{tabular}{|c|c|c|c|c|c|}
\hline \multirow{2}{*}{$\begin{array}{l}\text { Age des } \\
\text { fascioles }\end{array}$} & \multirow{2}{*}{$\begin{array}{l}\text { Doses } \\
\mathrm{mg} / \mathrm{kg}\end{array}$} & \multirow{2}{*}{$\begin{array}{l}\text { Nombre de } \\
\text { bouvillons } \\
\text { craitês }\end{array}$} & \multicolumn{2}{|c|}{$\begin{array}{l}\text { Nombre de douves } \\
\text { observées à } 1 \text { 'autopsie }\end{array}$} & \multirow{2}{*}{ Efficacité } \\
\hline & & & Vivantes & Nortes & \\
\hline 42 jours & 30 & 1 & 30 & 0 & Nu1le \\
\hline 42 jours & 40 & 1 & 158 & o & Nulle \\
\hline 54 jours & 30 & 1 & $I 12$ & 0 & Nulle \\
\hline 54 jours & 15 & 1 & 216 & 0 & Nulle \\
\hline 72 jours & 10 & 1 & 232 & 0 & Nulle \\
\hline 72 jours & 15 & 1 & 253 & 0 & Nulle \\
\hline 85 jours & 15 & 1 & 0 & $\begin{array}{c}552 \\
16-24 \mathrm{~mm} \times 2-3 \mathrm{~mm}\end{array}$ & Totale \\
\hline
\end{tabular}


3. Fascioles adultes pondeuses

Sept sujets faiblement parasités ont reçu une dose unique $(7,5-10$ ou $15 \mathrm{mg} / \mathrm{kg})$ de médicament. Les résultats sont indiqués dans le tableau V.

TABLEAU $\mathrm{N}^{\circ} \mathrm{V}$

\begin{tabular}{|c|c|c|c|}
\hline Doses (mg $/ \mathrm{kg}$ ) & 7,5 (1) & $10(1)$ & $15(2)$ \\
\hline Nombre d'antmaux utilisés & 2 & 3 & 2 \\
\hline $\begin{array}{l}\text { Nombre d'animaux totalement } \\
\text { déparasitês }\end{array}$ & 2 & 3 & 2 \\
\hline $\begin{array}{l}\text { Nombre de Fascioles } \\
\text { vivantes à l'autopsie }\end{array}$ & 0 & 0 & 0 \\
\hline $\begin{array}{l}\text { Nombre de Fascioles } \\
\text { mortes à 1'autopsie }\end{array}$ & - & 49 & 1 \\
\hline $\begin{array}{l}\text { Nombre d'animaux présentant } \\
\text { des oeufs et des lësions à } \\
\text { 1'autopste }\end{array}$ & 2 & 1 & 1 \\
\hline $\begin{array}{l}\text { (1) témolns : série B } \\
\text { (2) témoins : sêrie A }\end{array}$ & & & \\
\hline
\end{tabular}

\section{Paramphistomidés (tabl. VI)}

A l'autopsie les deux animaux traités à 7,5 et à $10 \mathrm{mg} / \mathrm{kg}$ ne présentent aucun parasite 5 et 6 jours après le traitement.

TABLEAU $\mathrm{N}^{\mathrm{a}} \mathrm{VI}$

Moyenne du nombre d'oeufs au gramme de matières fécales

\begin{tabular}{|c|c|c|c|}
\hline $\begin{array}{c}\text { Doses } \\
\mathrm{mg} / \mathrm{kg}\end{array}$ & $\begin{array}{c}\text { Avant } \\
\text { traitement }\end{array}$ & $\begin{array}{c}\text { Après } \\
\text { traitement }\end{array}$ & $\begin{array}{c}\text { Dernier } \\
\text { jour }\end{array}$ \\
\hline 7,5 & 52 & 126 & 0 \\
10 & 2047 & 498 & 0 \\
\hline
\end{tabular}

Tëmoins : série B

\section{Discussion}

En milieu tropical l'oxyclozanide peut éliminer les fascioles (Fasciola gigantica) de sujets faiblement parasités et faire disparaître les Paramphistomidés de la panse à la dose unique de $7,5 \mathrm{mg} / \mathrm{kg}$. Il est totalement inactif, même à des doses plus élevées, sur Schistosoma bovis et Dicrocoelium hospes.

Sur les formes immatures de Fasciola gigantica les résultats sont nettement moins bons. En effet, la dose de $40 \mathrm{mg} / \mathrm{kg}$ est insuffisante pour détruire les parasites de 6 semaines et celle de $15 \mathrm{mg} / \mathrm{kg}$ pour éliminer ceux qui sont âgés de 72 jours.

\section{Schistosoma bovis}

Les résultats de l'intervention, aux doses de 7,5 et $10 \mathrm{mg} / \mathrm{kg}$, contrôlés à l'autopsie, sont indiqués dans le tableau VII.

TABLEAU $\mathrm{N}^{\circ} \mathrm{VII}$

\begin{tabular}{|l|c|c|}
\hline Doses (mg/kg) & 7,5 & 10 \\
\hline $\begin{array}{l}\text { Nombre d'animaux totalement } \\
\text { déparasités }\end{array}$ & 0 & 0 \\
\hline $\begin{array}{l}\text { Nombre total de parasites } \\
\text { encore vivants à l'autopsie }\end{array}$ & 46 & 75 \\
\hline Efficacitè & Nulle & Nulle \\
\hline Tẻmoins : sêrie B & & \\
\hline
\end{tabular}

Si l'on compare les résultats obtenus, au Tchad, sur les bovins porteurs de fascioles à ceux qui ont été publiés dans divers pays, ils apparaissent très semblables. La plupart des auteurs reconnaissent en effet que la dose de $10 \mathrm{mg} / \mathrm{kg}$ peut éliminer 76 à 100 p. 100 des fascioles hépatiques (WALLEY, KELSEY, JONES (1966), FROYD (1968-1969), alors que REUSS et BROZEIT (1968, en Allemagne et HADNER (1968) en Suède obtiennent, toutefois, des résultats moins favorables.

Les effets douvicides de l'oxyclozanide constatés au Tchad sur les immatures, sont également très sensiblement identiques à ceux qui 
ont été observés par quelques auteurs aussi bien sur Fasciola hepatica que sur Fasciola gigantica.

Résultats de WALLEY (1966) obtenus sur les bovins infestés expérimentalement avec des métacercaires de Fasciola hepatica (tabl. VIII).

TABLEAU $N^{\circ}$ III

\begin{tabular}{|l|c|c|c|c|c|c|}
\hline Age des douves & \multicolumn{3}{|c|}{3 semaines } & \multicolumn{3}{c|}{$6-7$ semaines } \\
\hline Doses (mg/kg) & 10 & 15 & 30 & 15 & 25 & 50 \\
\hline Efficacité (p.100) & 5 & 41 & 28 & 25 & 35 & 80 \\
\hline
\end{tabular}

D'après le même auteur et BORAY et collab. (1967), chez le mouton la dose thérapeutique est d'au moins $15 \mathrm{mg} / \mathrm{kg}$ contre les douves pondeuses alors que 89 p. 100 des immatures de 6 à 7 semaines sont éliminées avec la dose élevée et dangereuse de $60 \mathrm{mg} / \mathrm{kg}$. Ce dernier résultat a été confirmé par HILDEBRANDT et ILMOLELIAN (1968) en Tanzanie sur Fasciola gigantica immature.

\section{Action sur les Nématodes}

L'action de l'oxyclozanide sur Cooperia punctata, Cooperia pectinata, Bunostomum phebotomum, Haemoncus contortus et les filaires du péritoine, de l'aorte et du ligament cervical est nulle à $7,5,10$ et $15 \mathrm{mg} / \mathrm{kg}$.

Un petit nombre de Trichures et d'Esophagostomes sont expulsés avec des doses de 7,5 ou de $10 \mathrm{mg} / \mathrm{kg}$. Les résultats sont peu significatifs, car trop irréguliers. Le même phénomène avait déjà été observé par WALLEY (1966) (tabl. IX).

TABLEAU $\mathrm{N}^{\circ}$ IX

\begin{tabular}{|c|c|c|c|}
\hline Doses $\quad(\mathrm{mg} / \mathrm{kg})$ & 7,5 (1) & $10(1)$ & $15(2)$ \\
\hline \multicolumn{4}{|c|}{$\begin{array}{l}\text { Nombre total de parasites } \\
\text { êlimirés }\end{array}$} \\
\hline $\begin{array}{l}\text {-B. radiatum } \\
\text {-Buck. globulosa }\end{array}$ & $\begin{array}{l}1 \\
2\end{array}$ & 2 & $\begin{array}{l}0 \\
0\end{array}$ \\
\hline \multicolumn{4}{|c|}{$\begin{array}{l}\text { Nombre total de parasites } \\
\text { restants à } l^{\prime} \text { autopsie }\end{array}$} \\
\hline $\begin{array}{l}\text { - B. radiatzom } \\
\text { Buck. globuicosa }\end{array}$ & $\begin{array}{r}43 \\
0\end{array}$ & $\stackrel{0}{-}$ & $\begin{array}{l}10 \\
20\end{array}$ \\
\hline $\begin{array}{l}\text { (1) témoins : série B } \\
\text { (2) témoins : sêrie A }\end{array}$ & & & \\
\hline
\end{tabular}

\section{MODE D'ACTION}

L'oxyclozanide semble agir rapidement sur les fascioles adultes qui sont tuées en moins de 5 jours, mais leur élimination est beaucoup plus lente et les délais d'expulsion varient en fonction de la dose et des réactions individuelles des animaux (tableau $\mathbf{X}$ ).

TABLEAU $\mathrm{N}^{\circ} \mathrm{X}$

\begin{tabular}{|c|c|c|}
\hline $\begin{array}{c}\text { Doses } \\
\mathrm{mg} / \mathrm{kg}\end{array}$ & $\begin{array}{c}\text { Absence } \\
\text { de douves }\end{array}$ & $\begin{array}{c}\text { Douves } \\
\text { prësentes }\end{array}$ \\
\hline 7,5 & 6 et 10 jours & - \\
9 jours & 7 et 8 jours \\
\hline
\end{tabular}

Les douves mortes dans les canaux biliaires 7 à 8 jours après le traitement sont en extension, verdâtres, diaphanes. Elles renferment encore de nombreux cufs, mais on ignore s'ils sont capables d'incuber ou non pour ultérieurement contaminer les limnées.

\section{TOXICITE}

Les essais, aux doses élevées de 50 ou de $75 \mathrm{mg} / \mathrm{kg}$ n'ont concerné que deux jeunes animaux dont les réactions ont été comparées à celles des sujets traités avec de plus faibles doses $(7,5,10$ et $15 \mathrm{mg} / \mathrm{kg}$ ) (tableau XI). 
TABLEAU $N^{\circ} \mathrm{XI}$

\begin{tabular}{|c|c|c|}
\hline \multirow{2}{*}{$\begin{array}{c}\text { Doses } \\
\mathrm{mg} / \mathrm{kg}\end{array}$} & Bouvillons & Femelles âgëes \\
\hline & 0 sur 2 & $0 \operatorname{sur} 3$ \\
7,5 & - & $0 \operatorname{sur} 4$ \\
10 & $0 \operatorname{sur} 3$ & \\
1.5 & $0 \operatorname{sur} 1$ & \\
75 & 1 sur 1 & \\
\hline
\end{tabular}

L'oxyclozanide paraît assez bien supporté aux doses thérapeutiques. Jusqu'à $15 \mathrm{mg} / \mathrm{kg}$, le médicament n'entraîne que des réactions limitées : léger ramollissement des selles et augmentation du nombre de celles-ci, avec perte passagère de l'appétit.

A des doses élevées $(75 \mathrm{mg} / \mathrm{kg}$ ), quelques heures après le traitement, on observe de l'inappétence et une certaine prostration. L'animal a des difficultés à maintenir son équilibre, titube, se couche sur le côté, pour ne plus se relever. La respiration s'accélère et la diarrhée apparaît. La mort survient en deux jours.

Les lésions n'ont rien de spécifique: elles se manifestent sous la forme d'une inflammation aiguë de l'intestin, avec, parfois, des hémorragies.

Les résultats obtenus corroborent ceux de WALLEY (1966) qui, chez le bœuf, enregistre une certaine mortalité à $60 \mathrm{mg} / \mathrm{kg}$.

Il en est de même pour le mouton européen.

Le mouton africain semble plus résistant malgré l'apparition d'une violente diarrhée qui frappe 80 p. 100 des ovins qui reçoivent des doses de $60 \mathrm{mg} / \mathrm{kg}$ (HILDEBRANDT et ILMOLELIAN, 1968).

\section{CONCLUSION}

D'après les expériences que nous avons effectuées, au Tchad, avec un trop petit nombre d'animaux, jeunes et vieux, de l'espèce bovine (zébu) nous avons pu cependant recueillir quelques faits intéressants:

1. L'oxyclozanide (pentachloro 3, 3', 5, 5', 6-dihydroxy $2,2^{\prime}$, salicylanilide) possède un réel pouvoir fasciolicide qui s'exerce à doses nettement différentes selon que Fasciola gigantica est adulte ou immature;

2. Les faibles doses de 7,5 à $10 \mathrm{mg} / \mathrm{kg}$ peuvent éliminer la plupart des fascioles adultes et les Paramphistomidés de la panse;

3. Si la dose de $15 \mathrm{mg} / \mathrm{kg}$ paraît suffisante pour chasser les fascioles âgées de 70 jours, elle ne permet pas de détruire celles de 6 à 7 semaines qui résistent à la dose de $40 \mathrm{mg} / \mathrm{kg}$;

4. Les fascioles adultes sont rapidement tuées; mais les délais d'élimination sont variables et exigent, parfois, une semaine avec la dose de $10 \mathrm{mg} / \mathrm{kg}$;

5. L'oxyclozanide n'a aucune activité à l'égard de Schistosoma bovis et de Dicrocoelium hospes et sur la plupart des Nématodes gastro-intestinaux;

6. Les accidents mortels apparaissant aux doses de $60-75 \mathrm{mg} / \mathrm{kg}$, le médicament ne peut être utilisé qu'avec beaucoup de précautions contre les immatures de 6 à 7 semaines.

\section{SUMMARY}

Effect of salicylanilide chlorine compound on different trematodes, parasites of zebu cattle in tropical Africa

In Tchad (Fort-Lamy), Oxyclozanid (pentachloro 3, 3', 5, 5', 6 dihydroxy $2,2^{\prime}$ salicylanilid) used by mouth at doses included between 7,5 and $15 \mathrm{mg} / \mathrm{kg}$ is active on Fasciola gigantica and Paramphistomum sp. of forestomach.

For the young flukes six to seven weeks old, $40 \mathrm{mg} / \mathrm{kg}$ is not sufficient.

The medicament is inefficacious on Schistosoma bovis and Dicrocoelium hospes.

The first fatal accidents arise at $60-75 \mathrm{mg} / \mathrm{kg}$. 


\section{RESUMEN}

Acción de un derivado clorado de la salicylanilida sobre los tremátodos, parásitos de los bovinos, en medio tropical

Se mostró activo contra Fasciola gigantica adulta y los Paramfistomidos de la panza el Oxyclozanido (pentacloro $3,3^{\prime}, 5,5^{\prime}$, 6 dihidroxi $2,2^{\prime}$ salicylanilida) administrado per os en dosis única de 7,5 o mejor de 10 a $15 \mathrm{mg} / \mathrm{kg}$ en cebues machos y hembras, en Fort-Lamy (Tchad). Para destruir las fasciolas inmaduras de 6 o 7 semanas, la dosis de $40 \mathrm{mg} / \mathrm{kg}$ es todavia insuficiente.

Dicho producto no tiene ningún actividad para con Schistosoma bov's

y Dicrocoelium hospes.

Accidentes mortales occurren en dosis de unos 60 a $75 \mathrm{mg} / \mathrm{kg}$.

\section{BIBLIOGRAPHIE}

ARUNDEL (J.H.), "Recent advances in anthelmintics », Aust. vet. J., 1967, 43. 455-9.

BORAY (J. C.), « Standardization of techniques for pathological and anthelmintic studies with Fascrola spp. ", Int. conf. wld. Ass. adv. vet. Parasit., Hanovre 1963, 1964 : 34-45.

BORAY (J.C.), HAPPICH (F. A.) et ANDREWS (J.C.), "Comparative chemotherapeutical tests in sheep infected with immature and mature F. hepatica», Vet. Rec. 1967, 80 (6) : 218-24.

BORAY (J. C.) et HAPPICH (F. A.), "Standardized chemotherapeutical tests for immature and mature Fasciola hepatica infections in sheep ", Aust. vet. J. $1968,44(2): 72-78$.

BROOME (A. W.) et JONES (W. G.), «A new drug for the treatment of Fasciolasis in sheep and cattle $»$, Nature, London, 1966, 210 (5037): 744-45.

FROYD (D. G.), «Field trials with oxyclozanide. A new liverfluke remedy for sheep and cattle ", Brit. vet. J. 1968,124 (3) : 116-25.

FROYD (G.), «The efficacy of Oxyclozanide in heavy cattle $\%$. Vet. Rec. 1969, 85 (25) : 705-07.

HEDNER (S.), "Essais de traitement avec le B1levon $\mathbf{R}$ et l'oxyclozanide en cas de distomatose », Svensk. Veterinärtidn, 1968, 22.

HILDEBRANDT (J.) et ILMOLELIAN (L. L.), « Ef- ficacy of Zanil (oxyclozanide) against immature and mature stages of Fusciola gigantica in experumentally infested sheep »,Berl. Munsch. Tierázzll. Wschr. 1968, 81 (9): 178-80.

JONES (E. H.), " Fasciolasis and oxyclozanide », Vet. Rec. 1966,79 (23) : 716-17.

KELSEY (F. H.), "Observations on the use of «Zanil» against liverfluke disease in wester ross ", Vet. Rec. 1966, 78 (9) : 303-04.

REISS (L. L.) et BROZEIT (H. E.) "Moglichkeiten der grobflächigen leberegelbe Kämpfung », Tierarztl. Umsch. 1968, 23: 403-09.

SINCLAIR (K.B.), \& Recent advances in knowledge of pathogenesis and treatment of fascioliasis $"$, Vet. Rec. 1968, 83 (24): 609.

TARCZYNSKI (S.) et collab., «Therapeutic studies on fasciolasis in ruminants. I - Efficacy of Zanil (oxyclozanide) $»$, Medycyna Wet., 1969, 25 : 154-58.

VAUGHAN (J. J.), «Preliminary fjeld trials with oxyclozanide, a new fasciolicide », Vet. Rec. 1966, 79 (24) : $720-23$.

WALLEY (J.K.), "Zanil " oxyclozanide in the treatment of the liver fluke $F$. hepatica in sheep and cattle », Vet, Rec. 1966, 78 (8): 267-76. 